CONFORMAL GEOMETRY AND DYNAMICS

An Electronic Journal of the American Mathematical Society

Volume 15, Pages 50-63 (June 7, 2011)

S 1088-4173(2011)00225-6

\title{
SHAPES OF TETRAHEDRA WITH PRESCRIBED CONE ANGLES
}

\author{
AHTZIRI GONZÁLEZ AND JORGE L. LÓPEZ-LÓPEZ
}

\begin{abstract}
Given real numbers $4 \pi>\theta_{0} \geq \theta_{1} \geq \theta_{2} \geq \theta_{3}>0$ so that $\sum_{j=0}^{3} \theta_{j}=4 \pi$, we provide a detailed description of the space of flat metrics on the 2 -sphere with 4 conical points of cone angles $\theta_{0}, \theta_{1}, \theta_{2}, \theta_{3}$, endowed with a geometric structure arising from the area function.
\end{abstract}

\section{INTRODUCTION}

Using different techniques, Deligne and Mostow [2] and Thurston [9] studied the space of orientation-preserving similarity classes of flat metrics on the sphere $S^{2}$ with $n$ conical singularities of prescribed conical angles (see also [7, 10], and [3]). A geometric structure was imposed on this space using different versions of the area function viewed as a Hermitian form.

The simplest nontrivial examples of such spaces considered in [2] and [9] are the spaces $\mathcal{T}\left(\theta_{1}, \theta_{2}, \theta_{3}\right)$ of orientation-preserving similarity classes of flat metrics on the sphere $S^{2}$ with 4 conical singularities of prescribed conical angles $4 \pi>$ $\theta_{0}, \theta_{1}, \theta_{2}, \theta_{3}>0$ so that $\sum_{j=0}^{3} \theta_{j}=4 \pi$, but it is difficult to obtain a detailed description of these examples from the cited papers. Our main goal herein is to understand these examples quite concretely. Moreover, 9] does not address the case $\theta_{0} \geq 2 \pi$, which is merely sketched in [2, Section 13]. In this paper, all the possibilities for $\theta_{0}$ are explored. We also provide a description of the quotient of $\mathcal{T}\left(\theta_{1}, \theta_{2}, \theta_{3}\right)$ upon the action of relabeling conical singularities with the same cone angles. Dealing the particular case of the sphere $S^{2}$ with 4 conical singularities allows us to give simple proofs.

While preparing the manuscript, the authors were informed by MuciñoRaymundo that a description of the space of similarity classes of flat metrics on $S^{2}$ with 4 conical singularities of cone angles $\theta_{0}=\theta_{1}=4 \pi / 3$ and $\theta_{2}=\theta_{3}=2 \pi / 3$ was announced by Cruz-Cota in a talk in July, 2010 (see also [1]).

1.1. Terminology, notation and overview. We define a tetrahedron as a 2sphere with a flat metric having four conical singularities, the so-called vertices. Let $\theta_{k}>0(k=0,1,2,3)$ be the cone angles at the vertices. The cone angles must satisfy the so-called Gauss-Bonnet condition, namely $\theta_{0}+\theta_{1}+\theta_{2}+\theta_{3}=4 \pi$ (see 4, for example).

Received by the editors December 7, 2010.

2010 Mathematics Subject Classification. Primary 51M20; Secondary 58D17, 51M10, 51M25.

The study was partially supported by funding from the UMSNH (by means of a project of the CIC) and the SEP (by means of the Red Temática de Colaboración "Álgebra, topología y análisis"). 
A hexagon in the plane $\mathbb{C}$ is determined by a point $\left(z_{1}, \ldots, z_{6}\right) \in \mathbb{C}^{6}$ where $z_{1}, \ldots, z_{6}$ denote its consecutive vertices. Notice that degenerated and self-crossing hexagons are allowed. A polygon is called simple if it satisfies two conditions: it does not have repeated vertices, and the interior of any edge does not intersect to another edge.

Let $\theta_{1}, \theta_{2}, \theta_{3}$ be real numbers in the interval $(0,2 \pi)$. We denote $V\left(\theta_{1}, \theta_{2}, \theta_{3}\right)$ the 3 -dimensional subspace of $\mathbb{C}^{6}$ satisfying the constraint

$$
\begin{aligned}
& z_{1}-z_{2}=e^{i \theta_{1}}\left(z_{3}-z_{2}\right), \\
& z_{3}-z_{4}=e^{i \theta_{2}}\left(z_{5}-z_{4}\right), \\
& z_{5}-z_{6}=e^{i \theta_{3}}\left(z_{1}-z_{6}\right) ;
\end{aligned}
$$

that is, any element $Z \in V\left(\theta_{1}, \theta_{2}, \theta_{3}\right)$ has the form

$$
Z=\left(z_{1}, \frac{e^{i \theta_{1}} z_{3}-z_{1}}{e^{i \theta_{1}}-1}, z_{3}, \frac{e^{i \theta_{2}} z_{5}-z_{3}}{e^{i \theta_{2}}-1}, z_{5}, \frac{e^{i \theta_{3}} z_{1}-z_{5}}{e^{i \theta_{3}}-1}\right) .
$$

Given a hexagon $Z \in V\left(\theta_{1}, \theta_{2}, \theta_{3}\right)$ which is simple, a tetrahedron $\diamond(Z)$ is obtained by gluing the edges $\left[z_{2 k+1}, z_{2 k}\right]$ and $\left[z_{2 k-1}, z_{2 k}\right]$ with a rotation of angle $\theta_{k}$ around the point $z_{2 k}$. The resulting tetrahedron $\diamond(Z)$ has cone points of cone angles $\theta_{1}, \theta_{2}, \theta_{3}, \theta_{0}=4 \pi-\left(\theta_{1}+\theta_{2}+\theta_{3}\right)$. In Section 2 it is shown that any tetrahedron with angles $\theta_{1}, \theta_{2}, \theta_{3}, \theta_{0}=4 \pi-\left(\theta_{1}+\theta_{2}+\theta_{3}\right)$ can be obtained by gluing the sides of a hexagon $Z \in V\left(\theta_{1}, \theta_{2}, \theta_{3}\right) \cup V\left(\theta_{1}, \theta_{3}, \theta_{2}\right)$ which is simple, where $V\left(\theta_{1}, \theta_{3}, \theta_{2}\right)$ denotes the 3-dimensional subspace of $\mathbb{C}^{6}$ satisfying the constraint

$$
\begin{aligned}
& z_{1}-z_{2}=e^{i \theta_{1}}\left(z_{3}-z_{2}\right), \\
& z_{3}-z_{4}=e^{i \theta_{3}}\left(z_{5}-z_{4}\right), \\
& z_{5}-z_{6}=e^{i \theta_{2}}\left(z_{1}-z_{6}\right) .
\end{aligned}
$$

In order to parametrize the space of orientation-preserving similarity classes of tetrahedra we must consider the space of hexagons up to orientation-preserving similarity; that is, two hexagons $\left(z_{1}, \ldots, z_{6}\right)$ and $\left(w_{1}, \ldots, w_{6}\right)$ are identified if and only if there is a complex affine transformation $f(z)=a z+b$ of $\mathbb{C}, a \neq 0$, so that $f\left(z_{k}\right)=w_{k}$ for all $k=1, \ldots, 6$. Up to a translation, each class of hexagons has a representative $\left(z_{1}, \ldots, z_{6}\right)$ such that $z_{1}=0$. Therefore the space of orientation-preserving similarity classes of hexagons coming from tetrahedra with cone angles $\theta_{1}, \theta_{2}, \theta_{3}$ is a subset of $\mathbb{P}\left(V_{0}\left(\theta_{1}, \theta_{2}, \theta_{3}\right)\right) \cup \mathbb{P}\left(V_{0}\left(\theta_{1}, \theta_{3}, \theta_{2}\right)\right)$, where $\mathbb{P}\left(V_{0}\left(\theta_{j}, \theta_{k}, \theta_{l}\right)\right)$ denotes the complex projectivization of the 2 -dimensional vector space $V_{0}\left(\theta_{j}, \theta_{k}, \theta_{l}\right)=\left\{Z \in V\left(\theta_{j}, \theta_{k}, \theta_{l}\right): z_{1}=0\right\}$.

Let $\mathcal{T}\left(\theta_{1}, \theta_{2}, \theta_{3}\right)$ be the space of orientation-preserving similarity classes of tetrahedra with cone angles $\theta_{1}, \theta_{2}, \theta_{3}, \theta_{0}=4 \pi-\left(\theta_{1}+\theta_{2}+\theta_{3}\right)$ and labeled vertices. In Section 3 we shall construct triangular regions $\triangle\left(\theta_{1}, \theta_{2}, \theta_{3}\right)$ and $\triangle\left(\theta_{1}, \theta_{3}, \theta_{2}\right)$ contained in $\mathbb{P}\left(V_{0}\left(\theta_{1}, \theta_{2}, \theta_{3}\right)\right)$ and $\mathbb{P}\left(V_{0}\left(\theta_{1}, \theta_{3}, \theta_{2}\right)\right)$, respectively, and we shall see that $\mathcal{T}\left(\theta_{1}, \theta_{2}, \theta_{3}\right)$ is obtained by pasting both triangular regions along its boundary.

The area Hermitian product

$$
\left\langle\left(z_{1}, \ldots, z_{6}\right),\left(w_{1}, \ldots, w_{6}\right)\right\rangle_{\mathcal{A}}=\frac{i}{4} \sum_{k=1}^{6}\left(z_{k} \bar{w}_{k+1}-z_{k+1} \bar{w}_{k}\right)
$$

determines a geometric structure on $\mathbb{P}\left(V_{0}\left(\theta_{1}, \theta_{j}, \theta_{k}\right)\right)$, where $\{j, k\}=\{2,3\}$. The real number $\langle Z, Z\rangle_{\mathcal{A}}$ is equal to the signed area of the hexagon $Z$. In Section 4 we shall prove that $\triangle\left(\theta_{1}, \theta_{j}, \theta_{k}\right),\{j, k\}=\{2,3\}$, is in fact an actual triangle 
(i.e. its edges are geodesics) with respect to the geometric structure determined in $\mathbb{P}\left(V_{0}\left(\theta_{1}, \theta_{j}, \theta_{k}\right)\right)$ by the area Hermitian product, and the edges of the pair of triangles $\triangle\left(\theta_{1}, \theta_{2}, \theta_{3}\right)$ and $\triangle\left(\theta_{1}, \theta_{3}, \theta_{2}\right)$ are glued by isometries of this geometric structure to obtain the space $\mathcal{T}\left(\theta_{1}, \theta_{2}, \theta_{3}\right)$. So we shall conclude that $\mathcal{T}\left(\theta_{1}, \theta_{2}, \theta_{3}\right)$ is a geometric sphere with exactly 3 singularities (Theorem 13). When at least two cone angles are equal, vertices with equal cone angles can be interchanged by an orientation-preserving similarity. We also consider the quotient of the singular geometric sphere $\mathcal{T}\left(\theta_{1}, \theta_{2}, \theta_{3}\right)$ upon the action of relabeling vertices with the same cone angles, and we prove that the quotient is also a geometric sphere with exactly 3 singularities (Theorem 14).

Finally, we describe the labeling of vertices with whom we work. Given an oriented tetrahedron $\diamond$, let $v_{0}$ be a vertex with a cone angle which is greater than or equal to other three. For $p, q \in \diamond,[p, q]$ will be used henceforth to denote a fix length minimizing path from $p$ to $q$ (for the existence of length minimizing paths see [5, Section 1.12], for example). Choose length minimizing paths $\left[v_{0}, v_{k}\right]$, for $k=1,2,3$, where $v_{1}, v_{2}, v_{3}$ are the other vertices labeled so that the oriented path $\gamma=\left[v_{0}, v_{1}\right] \cup\left[v_{1}, v_{0}\right] \cup\left[v_{0}, v_{2}\right] \cup\left[v_{2}, v_{0}\right] \cup\left[v_{0}, v_{3}\right] \cup\left[v_{3}, v_{0}\right]$ is compatible with the orientation of the open disc $\diamond \backslash \gamma$; that is, if $\omega$ is the unit tangent vector of $\gamma$, and $i \omega$ is the normal vector such that the pair $(\omega, i \omega)$ is positively oriented, then $i \omega$ is incoming to $\diamond \backslash \gamma$. There are three possibilities to make that marking of vertices, but we will pick $v_{1}$ to be a vertex $v_{k}$ with a greater cone angle; that is, if $\theta_{k}$ denotes the cone angle at the vertex $v_{k}$ for $k=0,1,2,3$, we have $4 \pi>\theta_{0} \geq \theta_{1} \geq \theta_{2}, \theta_{3}>0$. In this way, the labels of vertices are uniquely determined when the cone angles are pairwise distinct.

Remark 1. $\theta_{2}+\theta_{3}=2 \pi$ implies $\theta_{1}=\theta_{2}=\theta_{3}=\theta_{4}=\pi$.

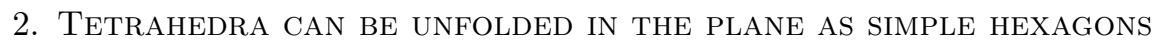

Lemma 2. (1) $\left[v_{0}, v_{j}\right] \cap\left[v_{0}, v_{k}\right]=\left\{v_{0}\right\}$ for $j \neq k$.

(2) Fix $k \in\{1,2,3\}$. At most two geodesic length minimizing paths from $v_{0}$ to $v_{k}$ may exist. If two of such paths exist, then there is exactly one geodesic length minimizing path from $v_{0}$ to $v_{j}$ for any $j \neq k$.

Proof. (1) Suppose $\left[v_{0}, v_{j}\right] \cap\left[v_{0}, v_{k}\right] \neq\left\{v_{0}\right\}$. Consideration of the local geometry around a vertex yields to observe that the geodesic path $\left[v_{0}, v_{k}\right]$ cannot visit a vertex $v_{l}$ of $\diamond$, unless $l \in\{0, k\}$. We take $p \in\left[v_{0}, v_{k}\right]$ to be the supreme of the set $\left[v_{0}, v_{j}\right] \cap\left[v_{0}, v_{k}\right]$. We have $p \in\left[v_{0}, v_{j}\right] \cap\left[v_{0}, v_{k}\right], p \neq v_{0}$ and $p$ is not a vertex of $\diamond$. The length of $\left[v_{0}, v_{k}\right]$ between $v_{0}$ and $p$ is equal to the length of $\left[v_{0}, v_{j}\right]$ between $v_{0}$ and $p$ because $\left[v_{0}, v_{j}\right]$ and $\left[v_{0}, v_{k}\right]$ are length minimizing paths. Consideration of the local flat metric around $p$ shows that there exist paths from $v_{0}$ to $v_{k}$ whose lengths are less than the length of $\left[v_{0}, v_{k}\right]$; a contradiction.

(2) Let $I$ and $J$ be two geodesic length minimizing paths from $v_{0}$ to $v_{k}$. By part (11) of Lemma 2, the union $I \cup J$ is a topological circle which separates $\diamond$ into two components. It is not difficult to see that each homotopy class of paths in $\diamond \backslash\left\{v_{0}, v_{1}, v_{2}, v_{3}\right\}$ has at most one geodesic representative (see [5, Section 1.13]). The result follows easily from this property and part (1) of Lemma 2 . 
The purpose of this section is to prove that any tetrahedron can be unfolded in the plane:

Proposition 3. There is an orientation-preserving isometric embedding $\Phi: \diamond \backslash$ $\gamma \rightarrow \mathbb{C}$ of the disk $\diamond \backslash \gamma$ into $\mathbb{C}$, both equipped with its Euclidean metric. The boundary $\partial(\Phi(\diamond \backslash \gamma))$ is a simple hexagon.

Such a map is called a developing map (see [8, Section 3.4]). We obtain Proposition 3 as an immediate consequence of Lemma 4.

Lemma 4. Let $\tilde{\Phi}: \diamond \rightarrow \mathbb{C}$ be the multivalued extension of the developing map $\Phi: \diamond \backslash \gamma \rightarrow \mathbb{C}$ that arises by continuity. Then $\tilde{\Phi}(p) \neq \tilde{\Phi}(q)$ for any pair of different points $p, q \in \gamma$.

Proof. We will obtain a contradiction supposing that $p, q \in \gamma$ are different points so that $\tilde{\Phi}(p)=\tilde{\Phi}(q)$. For such points $p, q$ with $\tilde{\Phi}(p)=\tilde{\Phi}(q)$, we have $p \in\left[v_{0}, v_{j}\right]$ and $q \in\left[v_{0}, v_{k}\right]$ where $j \neq k$ because $\tilde{\Phi}$ maps injectively geodesics into geodesics. Let $\alpha:[0,1] \rightarrow \diamond$ be a length minimizing path from $p$ to $q$. The intersection of $\gamma$ with the image $\alpha(0,1)$ of the open interval $(0,1)$ is nonempty because $\tilde{\Phi}$ maps injectively geodesics into geodesics. Moreover, $\gamma \cap \alpha(0,1)$ consists exactly of one point because otherwise there is $l \in\{1,2,3\}$ such that $\left[v_{0}, v_{l}\right] \cap \alpha([0,1])$ has two points, but this cannot happen by the proof of part (11) of Lemma 2. Therefore $\gamma \cap \alpha(0,1)=\{r\}$ where $r \in\left[v_{0}, v_{l}\right]$ with $l \neq j, k$. Let us suppose $r=\alpha\left(t_{0}\right)$. Replacing a small enough portion $\left[\alpha\left(t_{0}-\epsilon\right), \alpha\left(t_{0}+\epsilon\right)\right]$ of $\alpha[0,1]$ by a wedge shaped polygonal $\left[\alpha\left(t_{0}-\epsilon\right), u\right] \cup$ $\left[u, \alpha\left(t_{0}+\epsilon\right)\right]$ with $u$ close enough to the vertex $v_{l}$, we construct a polygonal path $\mathcal{L}=\left[p, \alpha\left(t_{0}-\epsilon\right)\right] \cup\left[\alpha\left(t_{0}-\epsilon\right), u\right] \cup\left[u, \alpha\left(t_{0}+\epsilon\right)\right] \cup\left[\alpha\left(t_{0}+\epsilon\right), q\right] \subset \diamond \backslash \gamma$ close to the polygonal path $\left[p, \alpha\left(t_{0}\right)\right] \cup\left[\alpha\left(t_{0}\right), v_{l}\right] \cup\left[v_{l}, \alpha\left(t_{0}\right)\right] \cup\left[\alpha\left(t_{0}\right), q\right] \subset\left[v_{0}, v_{l}\right] \cup \alpha[0,1]$. Since $\tilde{\Phi}(p)=\tilde{\Phi}(q)$ we may assume that $\tilde{\Phi}(\mathcal{L})$ is a simple polygon in $\mathbb{C}$. Otherwise we apply the argument to another polygonal path $\mathcal{L}^{\prime} \subset \mathcal{L}$ which $\tilde{\Phi}\left(\mathcal{L}^{\prime}\right)$ is a simple polygon. Hence, on the one hand, the sum of the exterior angles of $\tilde{\Phi}(\mathcal{L})$ should be equal to $\pm 2 \pi$ by part (11) of Lemma 16. On the other hand, the sum of the exterior angles of $\tilde{\Phi}(\mathcal{L})$ should be equal to 0 by part (2) of Lemma 16 because $\Phi$ is angle-preserving and it injectively maps geodesics into geodesics. This is a contradiction.

\section{Description of the SPACE $\mathcal{T}\left(\theta_{1}, \theta_{2}, \theta_{3}\right)$}

By Lemma 4, we can view $\tilde{\Phi}(\gamma)$ as a simple hexagon. Let us enumerate the vertices of $\tilde{\Phi}(\gamma)$ so that $\tilde{\Phi}\left(v_{1}\right)=z_{2}$. Since $\tilde{\Phi}(\gamma)$ is simple and positively oriented, the vertex $z_{2}$ uniquely determines the enumeration of the other vertices of $\tilde{\Phi}(\gamma)$. Therefore $Z=\left(z_{1}, z_{2}, z_{3}, z_{4}, z_{5}, z_{6}\right) \in V\left(\theta_{1}, \theta_{2}, \theta_{3}\right) \cup V\left(\theta_{1}, \theta_{3}, \theta_{2}\right)$. Up to an orientation-preserving similarity, $Z$ either is equivalent to

$$
\left(0, \frac{e^{i \theta_{1}}}{e^{i \theta_{1}}-1}, 1, \frac{e^{i \theta_{2}} \zeta-1}{e^{i \theta_{2}}-1}, \zeta, \frac{-\zeta}{e^{i \theta_{3}}-1}\right) \in V_{0}\left(\theta_{1}, \theta_{2}, \theta_{3}\right)
$$

when $Z$ is of the form of equation (1.1), or it is equivalent to

$$
\left(0, \frac{e^{i \theta_{1}}}{e^{i \theta_{1}}-1}, 1, \frac{e^{i \theta_{3}} \zeta-1}{e^{i \theta_{3}}-1}, \zeta, \frac{-\zeta}{e^{i \theta_{2}}-1}\right) \in V_{0}\left(\theta_{1}, \theta_{3}, \theta_{2}\right)
$$

when $Z \in V\left(\theta_{1}, \theta_{3}, \theta_{2}\right)$. 
Define $\triangle\left(\theta_{1}, \theta_{2}, \theta_{3}\right) \subset \mathbb{C}$ to be the triangular region

$$
\triangle\left(\theta_{1}, \theta_{2}, \theta_{3}\right)=\left\{\begin{array}{ll} 
& y \geq 0 \\
\zeta=x+i y: & x \sin \left(\theta_{2} / 2\right)+y \cos \left(\theta_{2} / 2\right) \geq 0 \\
& (1-x) \sin \left(\theta_{3} / 2\right)+y \cos \left(\theta_{3} / 2\right) \geq 0 \\
& \left(x-\frac{1}{2}\right)^{2}+\left(y+\frac{1}{2} \cot \left(\theta_{1} / 2\right)\right)^{2} \geq \frac{1}{4} \csc ^{2}\left(\theta_{1} / 2\right)
\end{array}\right\} .
$$

Here

- $x \sin \left(\theta_{2} / 2\right)+y \cos \left(\theta_{2} / 2\right)=0$ is a line through $(0,0)$ and angle $-\theta_{2} / 2$ with the positive $x$-axis.

- $(1-x) \sin \left(\theta_{3} / 2\right)+y \cos \left(\theta_{3} / 2\right)=0$ is a line through $(1,0)$ and angle $\theta_{3} / 2$ with the positive $x$-axis.

- $\left(x-\frac{1}{2}\right)^{2}+\left(y+\frac{1}{2} \cot \left(\theta_{1} / 2\right)\right)^{2}=\frac{1}{4} \csc ^{2}\left(\theta_{1} / 2\right)$ is a circle through $(0,0)$ and $(1,0)$.

Similarly, we define

$$
\triangle\left(\theta_{1}, \theta_{3}, \theta_{2}\right)=\left\{\begin{array}{ll} 
& y \geq 0 \\
\zeta=x+i y: & x \sin \left(\theta_{3} / 2\right)+y \cos \left(\theta_{3} / 2\right) \geq 0, \\
& (1-x) \sin \left(\theta_{2} / 2\right)+y \cos \left(\theta_{2} / 2\right) \geq 0, \\
& \left(x-\frac{1}{2}\right)^{2}+\left(y+\frac{1}{2} \cot \left(\theta_{1} / 2\right)\right)^{2} \geq \frac{1}{4} \csc ^{2}\left(\theta_{1} / 2\right)
\end{array}\right\} .
$$

Lemma 5. (1) Each point on the boundary of $\triangle\left(\theta_{1}, \theta_{2}, \theta_{3}\right)$, or on the boundary of $\triangle\left(\theta_{1}, \theta_{3}, \theta_{2}\right)$, encodes a tetrahedron $\diamond$ with two shortest paths from $v_{0}$ to another vertex.

(2) For each point on the boundary of $\triangle\left(\theta_{1}, \theta_{2}, \theta_{3}\right)$ there is exactly one point on the boundary of $\triangle\left(\theta_{1}, \theta_{3}, \theta_{2}\right)$ encoding the same tetrahedron.

Proof. (1) In the hexagon $\tilde{\Phi}(\gamma)$, the condition that a tetrahedron $\diamond$ has two shortest paths from $v_{0}$ to another vertex means one vertex $z_{2 k}$ has the same distance from $z_{1}, z_{3}, z_{5}$. Two of the segments $\left[z_{1}, z_{2 k}\right],\left[z_{3}, z_{2 k}\right]$, and $\left[z_{5}, z_{2 k}\right]$ are edges of the hexagon $\tilde{\Phi}(\gamma)$ and the other segment is interior to the hexagon $\tilde{\Phi}(\gamma)$.

Claim. If the hexagon $Z$ is normalized taking $z_{1}=0, z_{3}=1$ as in equation (3.1), and $z_{5}=\zeta=z+i y$, then

(a) $\left(x-\frac{1}{2}\right)^{2}+\left(y+\frac{1}{2} \cot \left(\theta_{1} / 2\right)\right)^{2}=\frac{1}{4} \csc ^{2}\left(\theta_{1} / 2\right)$ if and only if the segments $\left[z_{1}, z_{2}\right],\left[z_{3}, z_{2}\right]$, and $\left[z_{5}, z_{2}\right]$ have equal length,

(b) $x \sin \left(\theta_{2} / 2\right)+y \cos \left(\theta_{2} / 2\right)=0$ if and only if the segments $\left[z_{1}, z_{4}\right],\left[z_{3}, z_{4}\right]$, and $\left[z_{5}, z_{4}\right]$ have equal length,

(c) $(1-x) \sin \left(\theta_{3} / 2\right)+y \cos \left(\theta_{3} / 2\right)=0$ if and only if the segments $\left[z_{1}, z_{6}\right]$, $\left[z_{3}, z_{6}\right]$, and $\left[z_{5}, z_{6}\right]$ have equal length.

The Claim proves that each point on the boundary of $\triangle\left(\theta_{1}, \theta_{2}, \theta_{3}\right)$ encodes a tetrahedron $\diamond$ with two shortest paths from $v_{0}$ to another vertex. The proof for $\triangle\left(\theta_{1}, \theta_{3}, \theta_{2}\right)$ is similar.

Proof of the Claim. (a) The segments $\left[z_{1}, z_{2}\right],\left[z_{3}, z_{2}\right]$, and $\left[z_{5}, z_{2}\right]$ have equal length if and only if $z_{2}$ is center of a circle which pass through $z_{1}=0, z_{3}=1$ and $z_{5}=\zeta$. We have $z_{2}=\left(1-i \cot \left(\theta_{1} / 2\right)\right) / 2$ because $\angle z_{1} z_{2} z_{3}=\theta_{1}$. The equation of such a circle is $\left(x-\frac{1}{2}\right)^{2}+$ $\left(y+\frac{1}{2} \cot \left(\theta_{1} / 2\right)\right)^{2}=\frac{1}{4} \csc ^{2}\left(\theta_{1} / 2\right)$. 


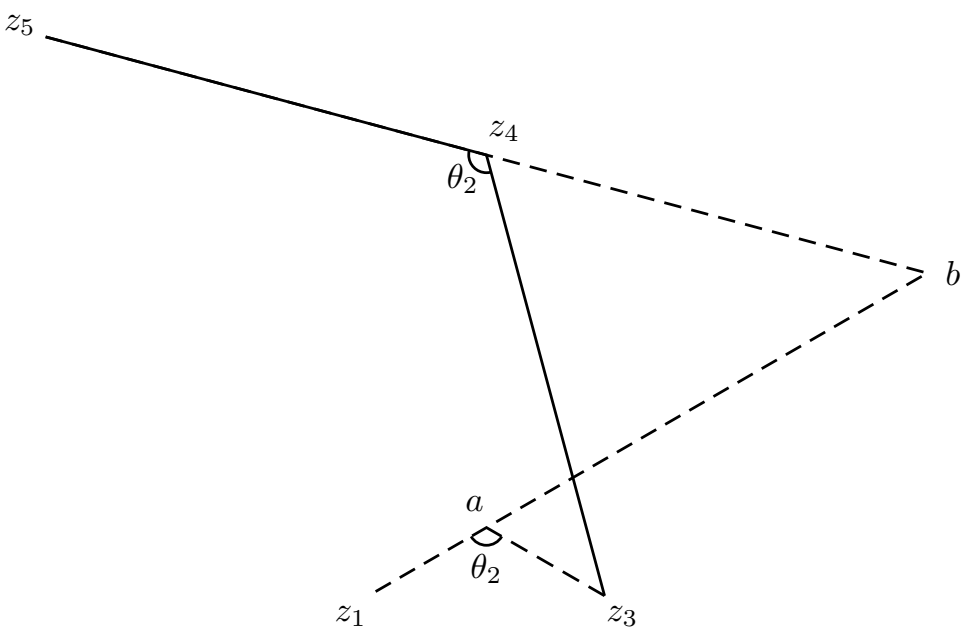

Figure 1. In the proof of the second part of the Claim in Lemma 5. we take $a$ in the upper half-plane for $\theta_{2}<\pi$, and $a$ in the lower half-plane for $\theta_{2}>\pi$. So the statements in the proof cover both cases.

(b) It is sufficient to prove that angle $\angle z_{3} z_{1} z_{5}=\pi-\theta_{2} / 2$ when the segments $\left[z_{1}, z_{4}\right],\left[z_{3}, z_{4}\right]$, and $\left[z_{5}, z_{4}\right]$ have equal length. Let $a$ be a point such that $\angle z_{1} a z_{3}=\theta_{2}$ and the segments $\left[z_{1}, a\right]$ and $\left[z_{3}, a\right]$ have equal length (see Figure 1). Let $b$ be the intersection of the line through $z_{1}$ and $a$ with the line through $z_{4}$ and $z_{5}$. Since $\angle z_{3} z_{1} b=\angle z_{3} z_{5} b$, then the quadrilateral $z_{1} z_{3} b z_{5}$ is cyclic and $\angle z_{5} b z_{1}=\angle z_{5} z_{3} z_{1}$. Since $\angle z_{5} z_{3} z_{1}=\angle z_{4} z_{3} a$, it follows that quadrilateral $a z_{3} b z_{4}$ is also cyclic. Therefore $\angle z_{3} z_{1} z_{5}=\pi-\angle z_{5} b z_{3}=\angle z_{3} a z_{4}=\pi-\theta_{2} / 2$.

(c) Is similar to the later case.

(2) Let $Z=\left(z_{1}, z_{2}, z_{3}, z_{4}, z_{5}, z_{6}\right)$ be a hexagon of the form of equation (3.1) such that $\left[z_{1}, z_{2 k}\right]=\left[z_{3}, z_{2 k}\right]=\left[z_{5}, z_{2 k}\right]$ for some $k \in\{1,2,3\}$. Hence $\zeta$ lies in the boundary of $\triangle\left(\theta_{1}, \theta_{2}, \theta_{3}\right)$. Two of the segments $\left[z_{1}, z_{2 k}\right],\left[z_{3}, z_{2 k}\right]$, and $\left[z_{5}, z_{2 k}\right]$ are edges of the hexagon $Z$ and the other is interior to the hexagon $Z$. If one cuts $Z$ along such interior segment and glues the two edges one obtains, after re-enumerating vertices in the appropriate way, a hexagon in $V\left(\theta_{1}, \theta_{3}, \theta_{2}\right)$ representing a point in the boundary of $\triangle\left(\theta_{1}, \theta_{3}, \theta_{2}\right)$.

We have proved the following corollary.

Corollary 6. $\mathcal{T}\left(\theta_{1}, \theta_{2}, \theta_{3}\right)$ is the disjoint union of $\triangle\left(\theta_{1}, \theta_{2}, \theta_{3}\right)$ and $\triangle\left(\theta_{1}, \theta_{3}, \theta_{2}\right)$ upon the identification of the boundaries $\partial \triangle\left(\theta_{1}, \theta_{2}, \theta_{3}\right)$ and $\partial \triangle\left(\theta_{1}, \theta_{3}, \theta_{2}\right)$ given by the reflection over the line $x=1 / 2$.

Remark 7. The corners of the triangular regions $\triangle\left(\theta_{1}, \theta_{2}, \theta_{3}\right)$ and $\triangle\left(\theta_{1}, \theta_{3}, \theta_{2}\right)$ encode the collision of two vertices whose cone angles add more than $2 \pi$ of the tetrahedron, namely,

- the corner of $\triangle\left(\theta_{1}, \theta_{2}, \theta_{3}\right)$ and $\triangle\left(\theta_{1}, \theta_{3}, \theta_{2}\right)$ between the sides $x \sin \left(\theta_{2} / 2\right)+$ $y \cos \left(\theta_{2} / 2\right)=0$ and $(1-x) \sin \left(\theta_{3} / 2\right)+y \cos \left(\theta_{3} / 2\right)=0$ encodes the collision between the vertices $v_{0}$ and $v_{1}$. 
- the other two corners of $\triangle\left(\theta_{1}, \theta_{2}, \theta_{3}\right)$ and $\triangle\left(\theta_{1}, \theta_{3}, \theta_{2}\right)$ encode the collision between an element of $\left\{v_{0}, v_{1}\right\}$ and an element of $\left\{v_{2}, v_{3}\right\}$.

\section{The geometry induced by the area Hermitian product}

4.1. The signature. Let $\langle\cdot, \cdot\rangle_{\mathcal{A}}$ be the area Hermitian product given by equation (1.2).

Proposition 8. The signature of the restriction of $\langle\cdot, \cdot\rangle_{\mathcal{A}}$ to the 2-dimensional vector spaces $V_{0}\left(\theta_{1}, \theta_{2}, \theta_{3}\right)$ and $V_{0}\left(\theta_{1}, \theta_{3}, \theta_{2}\right)$ is

$$
\begin{aligned}
& (0,2) \quad \text { for } \theta_{0}>2 \pi, \\
& (0,1) \quad \text { for } \theta_{0}=2 \pi, \\
& (1,1) \quad \text { for } \theta_{0}<2 \pi .
\end{aligned}
$$

This proposition is a particular case of [2, Corollaries 2.21, 13.1.2, 13.2.2] and [11, Theorem 14.3]. Also in [9, Proposition 3.3] a generalization of the case $\theta_{0}<2 \pi$ was proved. Consider that this particular case of tetrahedra allows us to give a different proof.

Proof. Two elements $Z_{0}, W_{0} \in V_{0}\left(\theta_{1}, \theta_{2}, \theta_{3}\right)$ are given by setting

$$
\begin{gathered}
W_{0}=\left(0,0,0, \frac{e^{i \theta_{2}}}{e^{i \theta_{2}}-1}, 1, \frac{-1}{e^{i \theta_{3}}-1}\right), \\
Z_{0}=\left(0, \frac{e^{i \theta_{1}}}{e^{i \theta_{1}}-1}, 1, \frac{e^{i \theta_{2}} \zeta-1}{e^{i \theta_{2}}-1}, \zeta, \frac{-\zeta}{e^{i \theta_{3}}-1}\right),
\end{gathered}
$$

where $\zeta=\frac{\sin \frac{\theta_{3}}{2}}{\sin \frac{\theta_{2}+\theta_{3}}{2}} e^{-i \theta_{2} / 2}$. We can easily compute

$$
\left\langle W_{0}, W_{0}\right\rangle_{\mathcal{A}}=\frac{\sin \frac{\theta_{2}+\theta_{3}}{2}}{4 \sin \frac{\theta_{2}}{2} \sin \frac{\theta_{3}}{2}}, \quad\left\langle Z_{0}, W_{0}\right\rangle_{\mathcal{A}}=0, \quad\left\langle Z_{0}, Z_{0}\right\rangle_{\mathcal{A}}=\frac{\sin \frac{\theta_{1}+\theta_{2}+\theta_{3}}{2}}{4 \sin \frac{\theta_{1}}{2} \sin \frac{\theta_{2}+\theta_{3}}{2}} .
$$

If $\left\langle W_{0}, W_{0}\right\rangle_{\mathcal{A}} \neq 0$ (i.e. $\theta_{2}+\theta_{3} \neq 2 \pi$ ), then we have, recalling that $\theta_{0}$ is a maximal angle,

$$
\begin{aligned}
& \left\langle W_{0}, W_{0}\right\rangle_{\mathcal{A}}>0 \text { and }\left\langle Z_{0}, Z_{0}\right\rangle_{\mathcal{A}}>0 \text { for } \theta_{1}+\theta_{2}+\theta_{3}<2 \pi, \\
& \left\langle W_{0}, W_{0}\right\rangle_{\mathcal{A}}>0 \text { and }\left\langle Z_{0}, Z_{0}\right\rangle_{\mathcal{A}}=0 \text { for } \theta_{1}+\theta_{2}+\theta_{3}=2 \pi, \\
& \left\langle W_{0}, W_{0}\right\rangle_{\mathcal{A}}>0 \text { and }\left\langle Z_{0}, Z_{0}\right\rangle_{\mathcal{A}}<0 \text { for } \theta_{1}+\theta_{2}+\theta_{3}>2 \pi .
\end{aligned}
$$

Since $\left\{W_{0}, Z_{0}\right\}$ is an orthogonal basis for the vector space $V_{0}\left(\theta_{1}, \theta_{2}, \theta_{3}\right)$, the proposition follows for the case $\theta_{2}+\theta_{3} \neq 2 \pi$.

If $\theta_{2}+\theta_{3}=2 \pi$, then $\theta_{1}=\theta_{2}=\theta_{3}=\theta_{4}=\pi$ (Remark 11). In this case we can choose equilateral triangles

$$
\begin{aligned}
W & =\left(1, \frac{1+\rho}{2}, \rho, \frac{\rho+\rho^{2}}{2}, \rho^{2}, \frac{1+\rho^{2}}{2}\right), \\
Z & =\left(1, \frac{1+\rho^{2}}{2}, \rho^{2}, \frac{\rho+\rho^{2}}{2}, \rho, \frac{1+\rho}{2}\right),
\end{aligned}
$$

where $\rho=e^{2 \pi i / 3}$. Then we have

$$
\langle W, W\rangle_{\mathcal{A}}=\frac{3 \sqrt{3}}{4}, \quad\langle W, Z\rangle_{\mathcal{A}}=0, \quad\langle Z, Z\rangle_{\mathcal{A}}=-\frac{3 \sqrt{3}}{4} .
$$

Next we translate the hexagons $w$ and $Z$ to get

$$
Z_{0}=Z-(1,1,1,1,1,1), W_{0}=W-(1,1,1,1,1,1) \in V_{0}\left(\theta_{1}, \theta_{2}, \theta_{3}\right) .
$$


It is easy to see that the area Hermitian product is invariant up to translations; that is

$$
\left\langle\left(z_{1}-\zeta, \ldots, z_{6}-\zeta\right),\left(w_{1}-\xi, \ldots, w_{6}-\xi\right)\right\rangle_{\mathcal{A}}=\left\langle\left(z_{1}, \ldots, z_{6}\right),\left(w_{1}, \ldots, w_{6}\right)\right\rangle_{\mathcal{A}}
$$

for all $\zeta, \xi \in \mathbb{C}$. Therefore $\left\{W_{0}, Z_{0}\right\}$ is an orthogonal basis for the vector space $V_{0}(\pi, \pi, \pi)$ and the proposition follows for the case $\theta_{2}+\theta_{3}=2 \pi$.

The proof for $V_{0}\left(\theta_{1}, \theta_{3}, \theta_{2}\right)$ is similar.

4.2. The geometric form. Now we recall how the projectivization of a Hermitian vector space can be geometrized. That is not another thing but the definition of the Bergman and Fubini-Study metrics.

Given $\theta_{1}, \theta_{2}, \theta_{3}$, we put $\mathcal{E}=\left\{Z \in V_{0}\left(\theta_{1}, \theta_{2}, \theta_{3}\right):\langle Z, Z\rangle_{\mathcal{A}}>0\right\}$. The complex projectivization $\mathbb{P}(\mathcal{E})$ can be seen as a space of hexagons with positive area up to equivalence by orientation-preserving similarities. By $\pi: \mathcal{E} \rightarrow \mathbb{P}(\mathcal{E})$ we denote the canonical projection. The Hermitian structure on $\mathcal{E}$ defined by

$$
\left\langle W_{1}, W_{2}\right\rangle_{Z}=\frac{\left\langle W_{1}, W_{2}\right\rangle_{\mathcal{A}}}{\langle Z, Z\rangle_{\mathcal{A}}}, \quad \text { for } Z \in \mathcal{E} \text { and } W_{1}, W_{2} \in T_{Z} \mathcal{E},
$$

is invariant up to multiplication by a complex scalar. We define on $\mathbb{P}(\mathcal{E})$ a pseudoHermitian structure $\langle\langle\cdot, \cdot\rangle\rangle$ so as to make the canonical projection $\pi: \mathcal{E} \rightarrow \mathbb{P}(\mathcal{E})$ a submersion (see [6, Example 1.11.14]): if $W_{1}, W_{2} \in T_{Z} \mathcal{E}$ we put $\left\langle\left\langle\pi_{*} W_{1}, \pi_{*} W_{2}\right\rangle\right\rangle_{\pi(Z)}$ $=\left\langle W_{1}, W_{2}\right\rangle_{Z}$. For each $Z \in \mathcal{E}$ so that $\langle Z, Z\rangle_{\mathcal{A}}=1$ one have a linear isomorphism onto $T_{\pi(Z)} \mathbb{P}(\mathcal{E})$ by restricting $\pi_{*}$ to the horizontal tangent subspace $T_{Z}^{h} \mathcal{E}=\{W \in$ $\left.T_{Z} \mathcal{E}:\langle W, Z\rangle_{\mathcal{A}}=0\right\}$. With this presentation of $T_{\pi(Z)} \mathbb{P}(\mathcal{E})$ one verifies that

$$
\pi_{*}(W)=\frac{1}{\langle Z, Z\rangle_{\mathcal{A}}^{1 / 2}}\left(W-\frac{\langle W, Z\rangle_{\mathcal{A}}}{\langle Z, Z\rangle_{\mathcal{A}}} Z\right)
$$

for any $Z \in \mathcal{E}$ and $W \in T_{Z} \mathcal{E}$. Hence,

$$
\left\langle\left\langle\pi_{*} W_{1}, \pi_{*} W_{2}\right\rangle\right\rangle_{\pi(Z)}=\frac{\left\langle W_{1}, W_{2}\right\rangle_{\mathcal{A}}\langle Z, Z\rangle_{\mathcal{A}}-\left\langle W_{1}, Z\right\rangle_{\mathcal{A}}\left\langle Z, W_{2}\right\rangle_{\mathcal{A}}}{\langle Z, Z\rangle_{\mathcal{A}}^{2}}
$$

for any $Z \in \mathcal{E}$ and $W_{1}, W_{2} \in T_{Z} \mathcal{E}$. Formula (4.1) also applies to $\mathbb{P}\left(\mathcal{E}^{\prime}\right)$, where $\mathcal{E}^{\prime}=\left\{Z \in V_{0}\left(\theta_{1}, \theta_{3}, \theta_{2}\right):\langle Z, Z\rangle_{\mathcal{A}}>0\right\}$.

Proposition 9. Endowed with the metric $\left\langle\langle\cdot, \cdot\rangle, \mathbb{P}(\mathcal{E})\right.$ and $\mathbb{P}\left(\mathcal{E}^{\prime}\right)$ are

(1) 2 -spheres of radius $1 / 2$ for $\theta_{0}>2 \pi$,

(2) planes with the zero metric for $\theta_{0}=2 \pi$,

(3) pseudo-Riemannian versions of the hyperbolic plane for $\theta_{0}<2 \pi$.

Proof. We prove the proposition for $\mathbb{P}(\mathcal{E})$. The proof for $\mathbb{P}\left(\mathcal{E}^{\prime}\right)$ is the same.

First suppose that $\theta_{0}>2 \pi$. The restriction of the area Hermitian product to $V_{0}\left(\theta_{1}, \theta_{2}, \theta_{3}\right)$ is given by $\left\langle\left(\zeta_{1}, \zeta_{2}\right),\left(\xi_{1}, \xi_{2}\right)\right\rangle_{\mathcal{A}}=\zeta_{1} \bar{\xi}_{1}+\zeta_{2} \bar{\xi}_{2}$ with respect to an $\langle\cdot, \cdot\rangle_{\mathcal{A}^{-}}$ orthonormal basis for $V_{0}\left(\theta_{1}, \theta_{2}, \theta_{3}\right)$ (Proposition 8 ). The projectivization $\mathbb{P}(\mathcal{E})$ is the complex projective line. If we take an affine chart $\left(\zeta_{1}: \zeta_{2}\right) \mapsto \zeta=\zeta_{2} / \zeta_{1}$ of $\mathbb{P}(\mathcal{E})$ and we regard the tangent vectors $\xi_{1}, \xi_{2} \in T_{\zeta} \mathbb{C}$ as the vectors $\left(0, \xi_{1}\right),\left(0, \xi_{2}\right) \in T_{(1, \zeta)} \mathcal{E}$ we get, applying the formula (4.1),

$$
\left\langle\left\langle\pi_{*}\left(0, \xi_{1}\right), \pi_{*}\left(0, \xi_{2}\right)\right\rangle\right\rangle_{\pi(1, \zeta)}=\frac{\xi_{1} \bar{\xi}_{2}}{\left(1+|\zeta|^{2}\right)^{2}} .
$$

This is the metric that comes from the stereographic projection from the north pole $(0,0,1)$ of the sphere $\left\{x^{2}+y^{2}+(z-1 / 2)^{2}=1 / 4\right\}$ onto the $x y$-plane. 
Now assume that $\theta_{0}=2 \pi$. There is a basis so that the restriction of the area Hermitian product to $V_{0}\left(\theta_{1}, \theta_{2}, \theta_{3}\right)$ takes the form $\left\langle\left(\zeta_{1}, \zeta_{2}\right),\left(\xi_{1}, \xi_{2}\right)\right\rangle_{\mathcal{A}}=\zeta_{2} \bar{\xi}_{2}$ (Proposition 8). The image of $\mathcal{E}$ under the affine chart $\left(\zeta_{1}: \zeta_{2}\right) \mapsto \zeta=\zeta_{2} / \zeta_{1}$ of $\mathbb{P}\left(V_{0}\left(\theta_{1}, \theta_{2}, \theta_{3}\right)\right)$ is the punctured plane $\mathbb{C} \backslash\{0\}$. We regard the tangent vectors $\xi_{1}, \xi_{2} \in T_{\zeta} \mathbb{C}$ as the vectors $\left(0, \xi_{1}\right),\left(0, \xi_{2}\right) \in T_{(1, \zeta)} \mathcal{E}$. Applying the formula (4.1) we get

$$
\left\langle\left\langle\pi_{*}\left(0, \xi_{1}\right), \pi_{*}\left(0, \xi_{2}\right)\right\rangle\right\rangle_{\pi(1, \zeta)}=0
$$

Finally suppose that $\theta_{0}<2 \pi$. The restriction of the area Hermitian product to $V_{0}\left(\theta_{1}, \theta_{2}, \theta_{3}\right)$ is given by $\left\langle\left(\zeta_{1}, \zeta_{2}\right),\left(\xi_{1}, \xi_{2}\right)\right\rangle_{\mathcal{A}}=-\zeta_{1} \bar{\xi}_{1}+\zeta_{2} \bar{\xi}_{2}$ with respect to an $\langle\cdot, \cdot\rangle_{\mathcal{A}}$-orthonormal basis for $V_{0}\left(\theta_{1}, \theta_{2}, \theta_{3}\right)$ (Proposition 8 ). The image of $\mathcal{E}$ under the affine chart $\left(\zeta_{1}: \zeta_{2}\right) \mapsto \zeta=\zeta_{2} / \zeta_{1}$ of $\mathbb{P}\left(V_{0}\left(\theta_{1}, \theta_{2}, \theta_{3}\right)\right)$ is $B=\{|\zeta|>1\}$. We regard the tangent vectors $\xi_{1}, \xi_{2} \in T_{\zeta} B$ as the vectors $\left(0, \xi_{1}\right),\left(0, \xi_{2}\right) \in T_{(1, \zeta)} \mathcal{E}$. Applying the formula (4.1) we get

$$
\left\langle\left\langle\pi_{*}\left(0, \xi_{1}\right), \pi_{*}\left(0, \xi_{2}\right)\right\rangle\right\rangle_{\pi(1, \zeta)}=-\frac{\xi_{1} \bar{\xi}_{2}}{\left(1-|\zeta|^{2}\right)^{2}} .
$$

Remark 10. Formula (4.3) determines a negative definite pseudo-Riemannian metric. In order to obtain a positive definite Riemannian metric, it is necessary to take the projectivization $\mathbb{P}(\mathcal{N})$ of hexagons with negative area $\mathcal{N}=\left\{Z \in V_{0}\left(\theta_{1}, \theta_{2}, \theta_{3}\right)\right.$ : $\left.\langle Z, Z\rangle_{\mathcal{A}}<0\right\}$, instead of taking $\mathbb{P}(\mathcal{E})$, to apply the formula (4.1) to get the positive definite hyperbolic metric $\frac{\xi_{1} \bar{\xi}_{2}}{\left(1-|\zeta|^{2}\right)^{2}}$ on the unitary ball $\left\{\left|\zeta_{2} / \zeta_{1}\right|<1\right\}$. Formula (4.1) changes to

$$
\left\langle\left\langle\pi_{*} W_{1}, \pi_{*} W_{2}\right\rangle\right\rangle_{\pi(Z)}=\frac{\left\langle W_{1}, Z\right\rangle_{\mathcal{A}}\left\langle Z, W_{2}\right\rangle_{\mathcal{A}}-\left\langle W_{1}, W_{2}\right\rangle_{\mathcal{A}}\langle Z, Z\rangle_{\mathcal{A}}}{\langle Z, Z\rangle_{\mathcal{A}}^{2}}
$$

and the positive definite hyperbolic metric $\frac{\xi_{1} \overline{\xi_{2}}}{\left(1-|\zeta|^{2}\right)^{2}}$ of constant curvature 4 on the unitary ball $\{|\zeta|<1\}$ is obtained.

Remark 11. Since

$$
V_{0}\left(\theta_{1}, \theta_{2}, \theta_{3}\right)=\left\{\left(0, \frac{e^{i \theta_{1}} \xi}{e^{i \theta_{1}}-1}, \xi, \frac{e^{i \theta_{2}} \zeta-\xi}{e^{i \theta_{2}}-1}, \zeta, \frac{-\zeta}{e^{i \theta_{3}}-1}\right): \xi, \zeta \in \mathbb{C}\right\}
$$

we may regard from now on the set

$$
\left\{\left(0, \frac{e^{i \theta_{1}}}{e^{i \theta_{1}}-1}, 1, \frac{e^{i \theta_{2}} \zeta-1}{e^{i \theta_{2}}-1}, \zeta, \frac{-\zeta}{e^{i \theta_{3}}-1}\right): \zeta \in \mathbb{C}\right\}
$$

as an affine chart of $\mathbb{P}\left(V_{0}\left(\theta_{1}, \theta_{2}, \theta_{3}\right)\right)$. In the proof of Proposition 8 we get an orthogonal basis $\left\{Z_{0}, W_{0}\right\}$ for $V_{0}\left(\theta_{1}, \theta_{2}, \theta_{3}\right)$. Notice that the line through $W_{0}$ is the point at infinity for the above affine chart (4.4). The normalizations of $Z_{0}$ and $W_{0}$ form an orthonormal basis for $V_{0}\left(\theta_{1}, \theta_{2}, \theta_{3}\right)$. Since any linear change of coordinates of a 2-dimensional vector space $V$ can be seen as a Möbius transformation of the projectivization $\mathbb{P}(V)$, we have that equation (4.1) determines in the above affine chart (4.4) a conformal model of the respective geometric model given in Proposition 9.

Lemma 12. If $\theta_{0} \neq 2 \pi$, then the boundary of $\triangle\left(\theta_{1}, \theta_{2}, \theta_{3}\right)$ and $\triangle\left(\theta_{1}, \theta_{3}, \theta_{2}\right)$ is composed by three geodesics of the corresponding geometric structure, and the angles between these geodesics are $\left|2 \pi-\theta_{1}-\theta_{2}\right| / 2,\left|2 \pi-\theta_{2}-\theta_{3}\right| / 2,\left|2 \pi-\theta_{3}-\theta_{1}\right| / 2$. 
Proof. We prove the lemma for $\triangle\left(\theta_{1}, \theta_{2}, \theta_{3}\right)$ with $\theta_{2}+\theta_{3} \neq 2 \pi$. The proofs for $\triangle\left(\theta_{1}, \theta_{3}, \theta_{2}\right)$ and $\triangle(\pi, \pi, \pi)$ are similar.

Case $\theta_{0}>2 \pi$. Recall the deduction of equation (4.2). The geodesic paths of the sphere $\left\{x^{2}+y^{2}+(z-1 / 2)^{2}=1 / 4\right\}$ are circles through antipodal points. If the $x y$-plane is viewed as the $\mathbb{C}$-plane, the points $\zeta_{1}$ and $\zeta_{2}$ represent antipodal points if and only if $\zeta_{2}=-1 / \bar{\zeta}_{1}$. Therefore two points $Z$ and $W$ in the affine chart (4.4) represent antipodal points in $\mathbb{P}\left(V_{0}\left(\theta_{1}, \theta_{2}, \theta_{3}\right)\right)$ if and only if $\langle Z, W\rangle_{\mathcal{A}}=0$.

Both lines $x \sin \left(\theta_{2} / 2\right)+y \cos \left(\theta_{2} / 2\right)=0$ and $(1-x) \sin \left(\theta_{3} / 2\right)+y \cos \left(\theta_{3} / 2\right)=0$ passing through the antipodal points

$$
\left(0,0,0, \frac{e^{i \theta_{2}}}{e^{i \theta_{2}}-1}, 1, \frac{-1}{e^{i \theta_{3}}-1}\right), \quad\left(0, \frac{e^{i \theta_{1}}}{e^{i \theta_{1}}-1}, 1, \frac{e^{i \theta_{2}} \zeta-1}{e^{i \theta_{2}}-1}, \zeta, \frac{-\zeta}{e^{i \theta_{3}}-1}\right),
$$

for $\zeta=\frac{\sin \frac{\theta_{3}}{2}}{\sin \frac{\theta_{2}+\theta_{3}}{2}} e^{-i \theta_{2} / 2}$. Since the metric is conformal (Remark 11), both lines represent circles in the sphere and the angle between them is equal to $\pi-\left(\theta_{2}+\theta_{3}\right) / 2$.

$\left(x-\frac{1}{2}\right)^{2}+\left(y+\frac{1}{2} \cot \left(\theta_{1} / 2\right)\right)^{2}=\frac{1}{4} \csc ^{2}\left(\theta_{1} / 2\right)$ passing through the antipodal points

$$
\left(0, \frac{e^{i \theta_{1}}}{e^{i \theta_{1}}-1}, 1, \frac{-1}{e^{i \theta_{2}}-1}, 0,0\right), \quad\left(0, \frac{e^{i \theta_{1}}}{e^{i \theta_{1}}-1}, 1, \frac{e^{i \theta_{2}} \zeta-1}{e^{i \theta_{2}}-1}, \zeta, \frac{-\zeta}{e^{i \theta_{3}}-1}\right)
$$

where $\zeta=\frac{\sin \frac{\theta_{1}+\theta_{2}}{2}}{\sin \frac{\theta_{1}}{2}} e^{-i \theta_{2} / 2}$. Since the metric is conformal (Remark 11), this circle represents a circle in the sphere forming angles equal to $\left|2 \pi-\theta_{1}-\theta_{2}\right| / 2$ and $\left|2 \pi-\theta_{3}-\theta_{1}\right| / 2$ with $x \sin \left(\theta_{2} / 2\right)+y \cos \left(\theta_{2} / 2\right)=0$ and $(1-x) \sin \left(\theta_{3} / 2\right)+y \cos \left(\theta_{3} / 2\right)=$ 0 , respectively.

Case $\theta_{0}<2 \pi$. The geodesics of the metric given by equation (4.3) are arcs of circle orthogonal to the unit circle because it can be seen as a conformal model of the hyperbolic geometry (Remarks 10 and 11). The boundary of $\mathcal{E} \subset V_{0}\left(\theta_{1}, \theta_{2}, \theta_{3}\right)$ is the set $\left\{Z \in V_{0}\left(\theta_{1}, \theta_{2}, \theta_{3}\right):\langle Z, Z\rangle_{\mathcal{A}}=0\right\}$. For $Z$ of the form

$$
Z=\left(0, \frac{e^{i \theta_{1}}}{e^{i \theta_{1}}-1}, 1, \frac{e^{i \theta_{2}} \zeta-1}{e^{i \theta_{2}}-1}, \zeta, \frac{-\zeta}{e^{i \theta_{3}}-1}\right)
$$

equation $\langle Z, Z\rangle_{\mathcal{A}}=0$ can be rewritten as

$$
\left(x-\frac{\cos \frac{\theta_{2}}{2} \sin \frac{\theta_{3}}{2}}{\sin \left(\frac{\theta_{2}+\theta_{3}}{2}\right)}\right)^{2}+\left(y+\frac{\sin \frac{\theta_{2}}{2} \sin \frac{\theta_{3}}{2}}{\sin \left(\frac{\theta_{2}+\theta_{3}}{2}\right)}\right)^{2}=-\frac{\sin \frac{\theta_{2}}{2} \sin \frac{\theta_{3}}{2} \sin \left(\frac{\theta_{1}+\theta_{2}+\theta_{3}}{2}\right)}{\sin \frac{\theta_{1}}{2} \sin ^{2}\left(\frac{\theta_{2}+\theta_{3}}{2}\right)}
$$

for $\zeta=x+i y$. This is a circle orthogonal to the paths

$$
\begin{gathered}
x \sin \left(\theta_{2} / 2\right)+y \cos \left(\theta_{2} / 2\right)=0 \\
(1-x) \sin \left(\theta_{3} / 2\right)+y \cos \left(\theta_{3} / 2\right)=0 \\
\left(x-\frac{1}{2}\right)^{2}+\left(y+\frac{1}{2} \cot \left(\theta_{1} / 2\right)\right)^{2}=\frac{1}{4} \csc ^{2}\left(\theta_{1} / 2\right),
\end{gathered}
$$

which form the mentioned angles. 


\section{THE MAIN THEOREM}

Theorem 13. $\mathcal{T}\left(\theta_{1}, \theta_{2}, \theta_{3}\right)$ is a thrice punctured 2-sphere.

For $\theta_{0}>2 \pi$ : The area Hermitian product endows $\mathcal{T}\left(\theta_{1}, \theta_{2}, \theta_{3}\right)$ with a metric of constant curvature 4 . The completion of this punctured geometric sphere is a sphere with three conical singularities with cone angles $\left|2 \pi-\theta_{1}-\theta_{2}\right|$, $\left|2 \pi-\theta_{2}-\theta_{3}\right|$, and $\left|2 \pi-\theta_{3}-\theta_{1}\right|$.

For $\theta_{0}<2 \pi$ : The area Hermitian product endows $\mathcal{T}\left(\theta_{1}, \theta_{2}, \theta_{3}\right)$ with a metric of constant curvature -4 . The completion of this punctured geometric sphere is a sphere with three singularities which can be conical singularities or cusps. The singularities have cone angles $\left|2 \pi-\theta_{1}-\theta_{2}\right|,\left|2 \pi-\theta_{2}-\theta_{3}\right|$, and $\left|2 \pi-\theta_{3}-\theta_{1}\right|$. When $2 \pi-\theta_{j}-\theta_{k}=0$, the singularity is a cusp.

The collision of vertices with cone angle $\theta_{k}$ and $\theta_{l}$ gives rise to the conical point in $\mathcal{T}\left(\theta_{1}, \theta_{2}, \theta_{3}\right)$ with cone angle $\left|2 \pi-\theta_{k}-\theta_{l}\right|=\left|2 \pi-\theta_{i}-\theta_{j}\right|$, where $\{i, j, k, l\}=$ $\{0,1,2,3\}$.

For $\theta_{0}<2 \pi$, Theorem 13 can be deduced from [9, Proposition 3.5] (see also [7. Section 5]).

Proof. To get the theorem as a consequence of Corollary 6, Remark 7, Proposition 9 and Lemma 12, we only have to verify that edges of $\triangle\left(\theta_{1}, \theta_{2}, \theta_{3}\right)$ and $\triangle\left(\theta_{1}, \theta_{3}, \theta_{2}\right)$ are pasted by isometries.

Regions $\triangle\left(\theta_{1}, \theta_{2}, \theta_{3}\right)$ and $\triangle\left(\theta_{1}, \theta_{3}, \theta_{2}\right)$ are contained in the affine charts

$$
\begin{aligned}
& \left\{\left(0, \frac{e^{i \theta_{1}}}{e^{i \theta_{1}}-1}, 1, \frac{e^{i \theta_{2}} \zeta-1}{e^{i \theta_{2}}-1}, \zeta, \frac{-\zeta}{e^{i \theta_{3}}-1}\right): \zeta \in \mathbb{C}\right\} \\
& \left\{\left(0, \frac{e^{i \theta_{1}}}{e^{i \theta_{1}}-1}, 1, \frac{e^{i \theta_{3}} \zeta-1}{e^{i \theta_{3}}-1}, \zeta, \frac{-\zeta}{e^{i \theta_{2}}-1}\right): \zeta \in \mathbb{C}\right\}
\end{aligned}
$$

of $\mathbb{P}\left(V_{0}\left(\theta_{1}, \theta_{2}, \theta_{3}\right)\right)$ and $\mathbb{P}\left(V_{0}\left(\theta_{1}, \theta_{3}, \theta_{2}\right)\right)$, respectively. Written in these charts, the function $\phi_{1}: \mathbb{P}\left(V_{0}\left(\theta_{1}, \theta_{2}, \theta_{3}\right)\right) \rightarrow \mathbb{P}\left(V_{0}\left(\theta_{1}, \theta_{3}, \theta_{2}\right)\right)$ given by the translation

$$
\zeta \mapsto \zeta+\left(\sin \left(\theta_{2} / 2\right) e^{-i \theta_{3} / 2}-\sin \left(\theta_{3} / 2\right) e^{-i \theta_{2} / 2}\right) / \sin \left(\left(\theta_{2}+\theta_{3}\right) / 2\right)
$$

and the function $\phi_{2}: \mathbb{P}\left(V_{0}\left(\theta_{1}, \theta_{3}, \theta_{2}\right)\right) \rightarrow \mathbb{P}\left(V_{0}\left(\theta_{1}, \theta_{3}, \theta_{2}\right)\right)$ given by the reflection on the vertical line through the point $\sin \left(\theta_{2} / 2\right) e^{-i \theta_{3} / 2} / \sin \left(\left(\theta_{2}+\theta_{3}\right) / 2\right)$ are isometries. Here $\sin \left(\theta_{3} / 2\right) e^{-i \theta_{2} / 2} / \sin \left(\left(\theta_{2}+\theta_{3}\right) / 2\right)$ is the intersection of lines $x \sin \left(\theta_{2} / 2\right)+$ $y \cos \left(\theta_{2} / 2\right)=0$ and $(1-x) \sin \left(\theta_{3} / 2\right)+y \cos \left(\theta_{3} / 2\right)=0$ compounding the boundary of $\triangle\left(\theta_{1}, \theta_{2}, \theta_{3}\right)$; and $\sin \left(\theta_{2} / 2\right) e^{-i \theta_{3} / 2} / \sin \left(\left(\theta_{2}+\theta_{3}\right) / 2\right)$ is the intersection of lines $x \sin \left(\theta_{3} / 2\right)+y \cos \left(\theta_{3} / 2\right)=0$ and $(1-x) \sin \left(\theta_{2} / 2\right)+y \cos \left(\theta_{2} / 2\right)=0$ compounding the boundary of $\triangle\left(\theta_{1}, \theta_{3}, \theta_{2}\right)$. The isometry $\phi_{2} \circ \phi_{1}: \mathbb{P}\left(V_{0}\left(\theta_{1}, \theta_{2}, \theta_{3}\right)\right) \rightarrow$ $\mathbb{P}\left(V_{0}\left(\theta_{1}, \theta_{3}, \theta_{2}\right)\right)$ is a realization of the gluing of the edges of $\triangle\left(\theta_{1}, \theta_{2}, \theta_{3}\right)$ and $\triangle\left(\theta_{1}, \theta_{3}, \theta_{2}\right)$ to get $\mathcal{T}\left(\theta_{1}, \theta_{2}, \theta_{3}\right)$.

\section{Relabeling Vertices With the SAme CONE ANGle}

Now we focus on the space of tetrahedra $\mathcal{T}\left(\theta_{1}, \theta_{2}, \theta_{3}\right)$ when two or more cone angles of the tetrahedra are equal. Theorem 13 establishes that $\mathcal{T}\left(\theta_{1}, \theta_{2}, \theta_{3}\right)$ is a 2-sphere with a Riemannian metric of constant curvature \pm 4 which has conical singularities with cone angles $\Theta_{j}=\left|2 \pi-\theta_{k}-\theta_{l}\right|$, where $\{j, k, l\}=\{1,2,3\}$. Equality between cone angles of the tetrahedra implies equality between cone angles of the 
space of tetrahedra $\mathcal{T}\left(\theta_{1}, \theta_{2}, \theta_{3}\right)$, namely:

(1) $\theta_{j}=\theta_{k} \Rightarrow \Theta_{j}=\Theta_{k}$ for $j, k \in\{1,2,3\}$.

(2) $\theta_{0}=\theta_{j} \Rightarrow \Theta_{k}=\Theta_{l}$ for $\{j, k, l\}=\{1,2,3\}$.

It is clear that:

(1) Unless $\Theta_{j}=\Theta_{k}$ for some pair $j \neq k$, the orientation-preserving symmetry group of $\mathcal{T}\left(\theta_{1}, \theta_{2}, \theta_{3}\right)$ is trivial.

(2) When exactly two angles $\Theta_{j}$ and $\Theta_{k}$ are equal, the orientation-preserving symmetry group of $\mathcal{T}\left(\theta_{1}, \theta_{2}, \theta_{3}\right)$ is the order two group.

(3) When $\Theta_{1}=\Theta_{2}=\Theta_{3}$, the orientation-preserving symmetry group of $\mathcal{T}\left(\theta_{1}, \theta_{2}, \theta_{3}\right)$ is the dihedral group $D_{3}$.

Let $\mathcal{S}=\mathcal{S}\left(\theta_{0}, \theta_{1}, \theta_{2}, \theta_{3}\right)$ denote the space of orientation-preserving similarity classes of tetrahedra with cone angles $2 \pi>\theta_{0}, \theta_{1}, \theta_{2}, \theta_{3}>0$ without labeled vertices. Let $\operatorname{Sym}\left(\mathcal{T}\left(\theta_{1}, \theta_{2}, \theta_{3}\right)\right)$ be the orientation-preserving symmetry group of $\mathcal{T}\left(\theta_{1}, \theta_{2}, \theta_{3}\right)$.

Theorem 14. $\mathcal{S}$ is the quotient $\mathcal{T}\left(\theta_{1}, \theta_{2}, \theta_{3}\right) / \operatorname{Sym}\left(\mathcal{T}\left(\theta_{1}, \theta_{2}, \theta_{3}\right)\right)$. It follows that $\mathcal{S}$ is a 2-sphere with a Riemannian metric of constant curvature \pm 4 which has three conical singularities.

(1) The tern of cone angles of $\mathcal{S}$ is $\left(\pi, \Theta_{j} / 2, \Theta_{k}\right)=\left(\pi, \Theta_{j} / 2, \Theta_{l}\right)$ when the angles $\theta_{k}$ and $\theta_{l}$ are the only ones that are equal, where $\{j, k, l\}=\{1,2,3\}$.

(2) The tern of cone angles of $\mathcal{S}$ is $\left(\pi, \Theta_{1} / 2, \Theta_{2}\right)=\left(\pi, \Theta_{1} / 2, \Theta_{3}\right)$ when either $\theta_{0}=\theta_{1} \neq \theta_{2}=\theta_{3}$ or the angles $\theta_{0}$ and $\theta_{1}$ are the only ones that are equal.

(3) The tern of cone angles of $\mathcal{S}$ is $\left(2 \pi / 3, \pi, \Theta_{1}\right)=\left(2 \pi / 3, \pi, \Theta_{2}\right)=\left(2 \pi / 3, \pi, \Theta_{3}\right)$ when at least three cone angles $\theta_{j}, \theta_{k}, \theta_{l}$ are equal, where $j, k, l \in\{0,1,2,3\}$.

Proof. On the one hand, it is easy to see that $\operatorname{Sym}\left(\mathcal{T}\left(\theta_{1}, \theta_{2}, \theta_{3}\right)\right)$ acts on $\mathcal{T}\left(\theta_{1}, \theta_{2}, \theta_{3}\right)$ identifying points which represent the same tetrahedron but with different labeled vertices.

On the other hand, the number of possible relabels of vertices with equal cone angle is $\left|\operatorname{Sym}\left(\mathcal{T}\left(\theta_{1}, \theta_{2}, \theta_{3}\right)\right)\right|$.

\section{Appendix A. Curvature of polygonal paths}

Let $Z=\left(z_{1}, \ldots, z_{n}\right)$ be a simple polygon. We called the interior of $Z$ to the set of points in the bounded component of the complement of $Z$. We said that the segment $[z, w]$ is contained in $Z$ if the interior of the segment $[z, w]$ is a subset of the interior of $Z$. A diagonal of $Z$ is a segment $\left[z_{j}, z_{k}\right]$ such that $k, j \in\{1, \ldots, n\}$ but $k \notin\{j, j \pm 1\}$.

Lemma 15. Let $Z=\left(z_{1}, \ldots, z_{n}\right)$ be a simple polygon with $n \geq 4$ vertices.

(1) $Z$ has a diagonal contained in $Z$.

(2) $Z$ admits a triangulation with $n-2$ triangles whose edges are of the form $\left[z_{j}, z_{k}\right]$, where $j, k \in\{1, \ldots, n\}$.

Proof. (1) Given $k \in\{2, \ldots, n-1\}$, the line through the points $z_{k-1}, z_{k}$ and the line through the points $z_{k}, z_{k+1}$ split together any ball centered at $z_{k}$ into four parts. Since the polygon is simple, one of these parts is contained in the interior of $Z$ for a small enough ball centered at $z_{k}$. Let $R_{0}$ be this small part contained in the interior of $Z$. Consider the maximal segments $\left[z_{k}, w\right]$ contained in $Z$ and intersecting $R_{0}$. If the ends $w$ of all these segments 
belong to the same edge of $Z$, then $\left[z_{k-1}, z_{k+1}\right]$ is a diagonal contained in $Z$; otherwise one of the segments $\left[z_{k}, w\right]$ is of the form $\left[z_{k}, z_{j}\right]$ with $j \neq k \pm 1$, and $\left[z_{k}, z_{j}\right]$ is a diagonal contained in $Z$.

(2) It can be proved by induction using part (11) of this lemma to reduce to polygons with a smaller number of vertices.

Let $Z=\left(z_{1}, \ldots, z_{n}\right)$ be a simple polygon. The term exterior angle to $Z$ at $z_{j}$ means the angle $-\pi<\alpha_{j} \leq \pi$ from the vector $z_{j}-z_{j-1}$ to the vector $z_{j+1}-z_{j}$ measured in the positive sense. In this way, the exterior angle at $z_{j}$ is the turning of the tangent vector at the vertex $z_{j}$.

A polygonal path in $\mathbb{C}$ is a union of segments $\bigcup_{j=1}^{n-1}\left[z_{j}, z_{j}+1\right]$. A polygonal path is said to be simple if it satisfies two conditions: it does not have repeated vertices, and the interior of any edge does not intersect to another edge. The term exterior angle of $\bigcup_{j=1}^{n-1}\left[z_{j}, z_{j}+1\right]$ at $z_{j}$, for $j \neq 1, n$, means the angle $-\pi<\alpha_{j} \leq \pi$ from the vector $z_{j}-z_{j-1}$ to the vector $z_{j+1}-z_{j}$ measured in the positive sense. By the exterior angle at $z_{n}$ we mean the angle $-\pi<\alpha_{n} \leq \pi$ from the vector $z_{n}-z_{n-1}$ to the vector $z_{2}-z_{1}$ measured in the positive sense. The definition is consistent with the definition of an exterior angle for a simple polygon (i.e. when $z_{n}=z_{1}$ ). By the sum of exterior angles we mean $\alpha_{2}+\alpha_{3}+\cdots+\alpha_{n}$.

Lemma 16. (1) Let $Z=\left(z_{1}, \ldots, z_{n}\right)$ be a simple polygon. The sum of exterior angles of $Z$ is equal to $2 \pi$ if $Z$ is positively oriented, and it is equal to $-2 \pi$ if $Z$ is negatively oriented.

(2) Let $L=\left[z_{1}, z_{2}\right] \cup\left[z_{2}, z_{3}\right] \cup\left[z_{3}, z_{4}\right] \cup\left[z_{4}, z_{5}\right]$ be a wedge shaped simple polygonal path with $\alpha_{5}=0$, like in Lemma 4, For any pair $s, t \in L$ the polygonal portion of $L$ which lies between $s$ and $t$ has the sum of exterior angles equal to 0 .

Proof. (1) By the interior angle of $Z$ at $z_{j}$ is meant $\theta_{j}=\pi-\alpha_{j}$. Notice that $0 \leq \theta_{j}<2 \pi$. From part (2) of Lemma 15] we have $\sum_{j=1}^{n} \theta_{j}=(n-2) \pi$. Therefore $\sum_{j=1}^{n} \alpha_{j}=2 \pi$ if $Z$ is positively oriented, and $\sum_{j=1}^{n} \alpha_{j}=-2 \pi$ if $Z$ is negatively oriented.

(2) There are several cases depending on which segments contain the points $s, t$. Each one of these cases is easy to verify.

\section{ACKNOWLEDGEMENTS}

The authors thank Aldo-Hilario Cruz-Cota for explaining his doctoral thesis and the preprint [1] to them. They are also grateful to Jesús Muciño-Raymundo for pointing out the work of Cruz-Cota. The authors gratefully acknowledge valuable and stimulating conversations with Alberto Verjovsky and Ferrán Valdez. The authors are grateful to Erika Atienzo for proof-reading the text.

\section{REFERENCES}

[1] A. H. Cruz-Cota, The moduli space of hex spheres, http://arxiv.org/abs/1010.5235, 2010.

[2] P. Deligne and G. D. Mostow, Monodromy of hypergeometric functions and non-lattice integral monodromy, Publ. Math. Inst. Hautes Études Sci. 63 (1986), 5-89. MR849651 (88a:22023a)

[3] F. Fillastre, From spaces of polygons to spaces of polyhedra following Bavard, Ghys and Thurston, http://fillastre.u-cergy.fr/articles.html, 2009. 
[4] H. Gluck, K. Krigelman, and D. Singer, The converse to the Gauss-Bonnet theorem in pl, J. Differential Geom. 9 (1974), 601-616. MR0390962 (52:11785)

[5] M. Gromov, Metric structures for Riemannian and non-Riemannian spaces, Birkhäuser, 1998. MR1699320(2000d:53065)

[6] W. Klingenberg, Riemannian geometry, de Gruyter Stud. Math., vol. 1, de Gruyter, 1982.

[7] S. Kojima, Complex hyperbolic cone structures on the configuration spaces, Rend. Istit. Mat. Univ. Trieste 32 (2001), 149-163. MR1893396 (2003b:55018)

[8] W. Thurston, Three-dimensional geometry and topology, vol. 1, Princeton University Press, 1997. MR 1435975 (97m:57016)

[9] Shapes of polyhedra and triangulations of the sphere, The Epstein Birthday Shrift, Geom. Topol. Monogr., vol. 1, 1998, pp. 511-549. MR.1668340 (2000b:57026)

[10] M. Troyanov, On the moduli space of singular Euclidean surfaces, Handbook of Teichmüller Theory, Vol. 1, IRMA Lect. Math. Theor. Phys., no. 11, 2007, pp. 507-540. MR2349679 (2009b:57041)

[11] W. A. Veech, Flat surfaces, Amer. J. Math. 115 (1993), no. 3, 589-689. MR.1221838 (94g:30043)

Cimat, Mineral de Valenciana, C.P. 36240, Guanajuato, Gto., Mexico

E-mail address: ahtziri@cimat.mx

Facultad de Ciencias Físico-matemáticas, UmSnh, Ciudad Universitaria, C.P. 58040, Morelia, Mich., Mexico

E-mail address: jllopez@umich.mx 